\title{
Defekte der Retina überschatten die Entwicklung
}

Fragestellung: Führt die Hemmung der beta-Sekretase BACE1 zu unerwünschten Nebeneffekten?

Hintergrund: BACE1 ist das Enzym, das zusammen mit der gamma-Sekretase aus dem Amyloid-Vorläuferprotein (APP) A-beta-Peptide freisetzt, die bei der Alzheimer-Demenz zum Verlust von Synapsen, zum Absterben von Neuronen und letztlich zum klinischen Bild der Krankheit führen. Daher wurde BACE1 als wertvolles Therapieziel identifiziert und Inhibitoren entwickelt, die sich zum Teil bereits in der klinischen Testung befinden (Übersicht z. B. in [1]). Neben APP kann BACE1 jedoch auch weitere Proteine prozessieren wie etwa Untereinheiten von Ionenkanälen, den Interleukin-1-Rezeptor oder den Myelinisierungsfaktor Neuregulin. Es ist daher nicht auszuschließen, dass eine Hemmung von BACE1 im Rahmen einer Alzheimer-Therapie zu Nebenwirkungen führen könnte. Die Entwicklung eines solchen Inhibitors wurde zum Beispiel vor kurzem eingestellt, da sich im Tiermodell Defekte an der Retina fanden [2].

Methodik: Die Retina von BACE1-Knock-Out-Mäusen wurde auf Vaskularisierung und Dicke hin untersucht. Ebenso wurden die Effekte einer intravitrealen Applikation eines BACE1-Inhibitors beziehungsweise einer siRNA gegen BACE1 analysiert. Die molekularen Mechanismen, über die eine BACE1-Hemmung zu einer Retinapathologie führen kann, wurden in Zellkulturversuchen mittels Western-Blot und Organellen-spezifischer Fluoreszenzmarker untersucht.

Ergebnisse: In der neuralen Retina von Mäusen wird BACE1 im Vergleich zum Gehirn wesentlich schwächer exprimiert (<50\%). Dennoch kommt es in Mäusen ohne BACE1 zu einer ausgeprägten Retinapathologie: Im Vergleich zum Wildtyp ist die Retinadicke um die Hälfte reduziert. Zudem finden sich atrophe retinale Ganglionzellen. Die atrophen Bereiche sind dabei mit einer Akkumulation des Alterspigmentes Lipofuscin korreliert. Eine Behandlung von primären humanen retinalen Pigmentepithelzellen ergab, dass eine Inhibition von BACE1 zu einer Veränderung der Lysosomen in den Zellen führt. Eine Färbung der retinalen Gefäße zeigte eine verminderte Kapillardichte in BACE1-Knock-Out-Mäusen im Vergleich zu WildtypTieren. Bei Applikation eines BACE1-Inhibitors oder einer spezifischen siRNA wurde die Neovaskularisierung nach Laserinduzierter Läsion des Auges in Mäusen vermindert. Da

Cai J, Qi X, Kociok $\mathrm{N}$ et al. $\beta$-Secretase (BACE1) inhibition causes retinal pathology by vascular dysregulation and accumulation of age pigment. EMBO Molecular Medicine 2012; 4, $1-12$ BACE1 die Spaltung von transmembranen Proteinen katalysiert, wurde eine mögliche Prozessierung des Rezeptors für den vaskulären endothelialen Wachstumsfaktor (VEGFR1) durch BACE1 in Zellkultur überprüft: Die Spaltung des VGFR1 wurde durch einen BACE1Inhibitor geblockt.

Schlussfolgerungen: Die Hemmung von BACE1 in Mäusen führt zu einer Retinapathologie. Es wurden zwei Mechanismen identifiziert, durch die eine veränderte BACE1-Aktivität die Retina beeinflusst: eine Dysregulation der VEGF-Signalweiterleitung und eine Abnahme der lysosomalen Funktion.

\section{- Kommentar von Dr. Kristina Endres \\ Spezifische Inhibitoren nötig}

Cai und Kollegen zeigen, dass eine Manipulation der betaSekretase BACE1 im Rahmen einer Alzheimer-Therapie möglicherweise mit Nebenwirkungen verbunden sein kann. Eine Medikation mit BACE1-Hemmern könnte zum Beispiel bei Patienten mit bereits bestehender Maculadegeneration zu einer weiteren Verschlechterung der Sehfähigkeit beitragen. Lipofuscin, das in BACE1-Knock-Out-Mäusen akkumulierte Alterspigment, findet sich zudem in Nervenzellen und ist bei der neuronalen Ceroid-Lipofuszinose mit dem Auftreten von Demenz assoziiert. Damit könnte eine Hemmung des BACE1 direkt zu neurodegenerativen Prozessen beitragen.

Auch für die gamma-Sekretase, ein weiteres Therapieziel, waren anfänglich starke Nebenwirkungen zu beobachten: Neben APP spaltet dieses Enzym unter anderem den NotchRezeptor, sodass beispielsweise Schädigungen des Gastrointestinaltrakts auftraten. Derzeit wird versucht, gamma-Sekretase-Modulatoren mit ausreichender APP-Spezifität zu finden, um die klinische Forschung weiter zu führen [3]. Ob dies auch für BACE1 gelingen kann, ist fraglich.

\footnotetext{
Referenzen

1. Mangialasche F et al. Lancet Neurol 2010; 9: 702-16

2. May PC et al. J Neurosci 2011; 31: 16507-16

3. D’Onofrio G et al. Expert Opin Drug Discov 2012; 7: 19-37
}

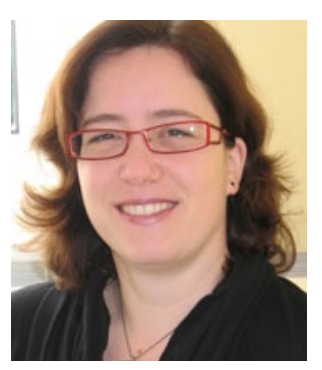

Dr. rer. nat. Kristina Endres, Mainz

Klinik für Psychiatrie und Psychotherapie, Universitätsmedizin Johannes GutenbergUniversität Mainz E-Mail: endres_k@psychiatrie.klinik.unimainz.de 